\title{
Relativistic RPA for Isobaric Analogue and Gamow-Teller Resonances in Closed Shell Nuclei
}

\author{
C. De Conti and A. P. Galeão \\ Instituto de Física Teórica, Universidade Estadual Paulista \\ Rua Pamplona 145, 01405.900 São Paulo, SP, Brazil \\ and \\ F. Krmpotić \\ Departamento de Física, Facultad de Ciencias Exactas \\ Universidad Nacional de La Plata \\ C.C. 671900 La Plata, Argentina
}

April 16, 2018

\begin{abstract}
We perform a self-consistent relativistic RPA calculation for the isobaric analogue and Gamow-Teller resonances based on relativistic mean field theory results for the ground states of ${ }^{48} \mathrm{Ca},{ }^{90} \mathrm{Zr}$ and ${ }^{208} \mathrm{~Pb}$. We use the parameter set NL1 for the $\sigma, \omega$ and $\rho$ mesons, and experimental values for the pion and nucleon. An extra parameter, related to the intensity of the contact term in the pion-exchange interaction, is crucial to reproduce the latter resonances.
\end{abstract}


In recent years the relativistic mean field theory (RMFT) has been successfully applied to account for both: i) the bulk features of nuclear matter (saturation, equation of state, etc.) and ii) the ground state properties of finite nuclei, including unstable ones up to the nucleon drip lines [1]. (For reviews, see refs. [2, 3, 4, 5].) A nice feature of the model is that the same restricted set of parameters can be used for all these systems. Even though some excited nuclear states, including certain giant resonances, have also been calculated in the relativistic random phase approximation (RRPA) based on RMFT, this has never been done for charge-exchange excitations. The aim of the present work is to explore to what extent this relativistic model, whose parameters have been fitted to ground state properties, can describe the charge-exchange collective states, such as the isobaric analogue state (IAS) and the Gamow-Teller resonance (GTR).

Both IAS and GTR have been extensively calculated in the context of traditional, i.e., nonrelativistic, nuclear structure theory, usually in the random phase approximation (RPA) or extensions of it, making use of, not only completely phenomenological interactions, but also more realistic ones based on meson exchange. While the IAS is easily reproduced, the GTR is more problematic, especially with respect to its strength that turns out to be quenched to $\sim 60 \%$ of the Ikeda sum-rule value. (For a review of GTR, see ref. [13].) Two mechanisms have been proposed for the absorption of the unseen strength: i) the excitation of the $\Delta$ resonance, which has the same quantum numbers as the GTR, or ii) the influence of $2 \mathrm{p}-2 \mathrm{~h}$ and higher excitations, not included in RPA. We shall not dwell on such finer points in this paper, since this would require, anyway, a more sophisticated treatment than the simple version of RRPA we are using, but rather will concentrate on the quality of the reproduction of excitation energy and strength as compared with similar nonrelativistic calculations [12, 14].

Relativistic mean field theory (RMFT) always takes as a starting point some version of an effective quantum field theory describing a system of interacting hadrons, coupled also to the electromagnetic (or to the electroweak) field, generally referred to as quantum hadrodynamics (QHD) [2, 3, 4, 5]. Historically, QHD was introduced as a renormalizable quantum field theory, which severely limited the number of admissible terms in its Lagrangian [2]. However, despite its many successes, it had difficulties with its renormalization program. Thus, in the last years, it began to be formulated as an effective, nonrenormalizable quantum field theory representing the low- 
energy limit of the fundamental theory of strong interactions, namely, quantum chromodynamics (QCD). Consequently, all terms compatible with the symmetries of QCD were now admissible, and one had to find appropriate expansion parameters and a criterion of naturalness to select the most important ones. Even thus, the number of terms increased significantly. Their coupling constants were adjusted to a judicious selection of nuclear properties that should be well reproduced at the mean field level and, while this was successful in the sense that they turned out to be natural, on the other hand there are clear indications that their full set is underdetermined [5]. Since our main purpose here is not to test the foundations of QHD, but rather to assess its ability to reproduce some new nuclear properties, and we want to keep the number of free parameters to a minimum, we will include only the most important terms, and work with the following nonrenormalizable QHD Lagrangian density:

$$
\begin{aligned}
\mathcal{L}=\bar{\psi} & \left(i \gamma_{\mu} \partial^{\mu}-M\right) \psi \\
& +\frac{1}{2} \partial_{\mu} \sigma \partial^{\mu} \sigma-\frac{1}{2} m_{\sigma}{ }^{2} \sigma^{2}-\frac{1}{3} g_{2} \sigma^{3}-\frac{1}{4} g_{3} \sigma^{4}-g_{\sigma} \bar{\psi} \psi \sigma \\
& -\frac{1}{4} \Omega_{\mu \nu} \Omega^{\mu \nu}+\frac{1}{2} m_{\omega}{ }^{2} \omega_{\mu} \omega^{\mu}-g_{\omega} \bar{\psi} \gamma_{\mu} \psi \omega^{\mu} \\
& +\frac{1}{2} \partial_{\mu} \boldsymbol{\pi} \cdot \partial^{\mu} \boldsymbol{\pi}-\frac{1}{2} m_{\pi}{ }^{2} \boldsymbol{\pi} \cdot \boldsymbol{\pi}-\frac{f_{\pi}}{m_{\pi}} \bar{\psi} \gamma_{5} \gamma_{\mu} \boldsymbol{\tau} \psi \cdot \partial^{\mu} \boldsymbol{\pi} \\
& -\frac{1}{4} \boldsymbol{R}_{\mu \nu} \cdot \boldsymbol{R}^{\mu \nu}+\frac{1}{2} m_{\rho}{ }^{2} \boldsymbol{\rho}_{\mu} \cdot \boldsymbol{\rho}^{\mu}-\frac{g_{\rho}}{2} \bar{\psi} \gamma_{\mu} \boldsymbol{\tau} \psi \cdot \boldsymbol{\rho}^{\mu} \\
& -\frac{1}{4} F_{\mu \nu} F^{\mu \nu}-e \bar{\psi} \gamma_{\mu} \frac{1+\tau_{3}}{2} \psi A^{\mu}
\end{aligned}
$$

in standard notation $(\hbar=c=1)$, where $\psi$ is the spin- $\frac{1}{2}$, isospin- $\frac{1}{2}$ nucleon field, $\sigma, \omega^{\mu}, \boldsymbol{\pi}$ and $\boldsymbol{\rho}^{\mu}$ denote the meson fields, and $A^{\mu}$ is the electromagnetic field [3]. The field tensors for the vector particles read

$$
\begin{aligned}
\Omega^{\mu \nu} & =\partial^{\mu} \omega^{\nu}-\partial^{\nu} \omega^{\mu} \\
\boldsymbol{R}^{\mu \nu} & =\partial^{\mu} \boldsymbol{\rho}^{\nu}-\partial^{\nu} \boldsymbol{\rho}^{\mu}-g_{\rho} \boldsymbol{\rho}^{\mu} \times \boldsymbol{\rho}^{\nu} \\
F^{\mu \nu} & =\partial^{\mu} A^{\nu}-\partial^{\nu} A^{\mu}
\end{aligned}
$$

To reproduce the experimental values of the nuclear incompressibility and surface diffuseness in RMFT, it has been found important [8] to allow the sigma meson to self-interact. This is done through the cubic and quartic 
terms, in $\sigma$, appearing in eq. (11). All couplings are taken to be of the direct, nonderivative kind, except in the pion-nucleon case, where both the phenomenology and theoretical reasons (related to chiral symmetry) definitively favor the pseudovector coupling. It is true that phenomenology would also recommend that a certain amount of derivative coupling should be mixed in for the vector mesons to the nucleon [11]. Yet, for simplicity, we followed the general trend in RMFT calculations [1, 7, 9] and did not include such terms in eq. (11). For the omega meson, this can be justified since that term would be rather small [10], but for the rho meson, while unimportant for the nuclear ground state, the corresponding term would give a sizable contribution to excitations of the Gamow-Teller type. Finally, the photon is minimally coupled to the proton to take care of the important Coulomb interaction inside nuclei, but not to the charged mesons since this would be of much less relevance for the properties of interest here and is hence ignored in this model Lagrangian.

The nucleon mass Đ, $M$, the proton charge, $e$, the pion mass, $m_{\pi}$, and coupling constant, $f_{\pi}$, are fixed at their experimental values. Thus, the masses of the remaining mesons included in eq. (1) $m_{\sigma}, m_{\omega}$ and $m_{\rho}$, their coupling constants, $g_{\sigma}, g_{\omega}$ and $g_{\rho}$, and the self-interaction strengths for the sigma meson, $g_{2}$ and $g_{3}$, give a total of only 8 free parameters for this model.

RMFT results from an approximation scheme to solve the Euler-Lagrange equations derived from $\mathcal{L}$, which consists in taking advantage of the high values of the densities inside nuclei to replace the meson and photon fields by classical fields satisfying these equations with the source terms replaced by their expectation values in the nuclear ground state of interest. This is equivalent to compute the meson self-energies in the Hartree approximation disregarding the negative-energy, i.e., antiparticle, states, which is sometimes referred to as the no-sea approximation.

The RMFT equations simplify considerably if one takes advantage of known symmetries of the nuclear ground state. Firstly, as it always has a definite parity, the pion, due to its pseudoscalar nature, completely disappears from these equations. Secondly, the definite charge of the nucleus eliminates all but the third component of the isovector mesons. Also, we are interested here only in spherical nuclei, and their rotational invariance implies that the spatial components of the currents in the source terms van-

\footnotetext{
${ }^{1}$ The proton mass is used here.
} 
ish in RMFT. So, the corresponding components of the vector fields can be ignored. Finally, we are looking only for stationary solutions in the nuclear rest frame. Thus, the meson and photon fields are taken as time-independent and the nucleon field has merely time-dependent phase factors, i.e.,

$$
\psi\left(x_{\mu}\right)=\sum_{\alpha} a_{\alpha} \mathcal{U}_{\alpha}(\boldsymbol{r}) \exp \left(-i E_{\alpha} t\right),
$$

where the no-sea approximation has been made, and $a_{\alpha}$ is the annihilation operator in the particle state with (positive) energy $E_{\alpha}$. With these simplifications, the RMFT equations become

$$
\left[-i \boldsymbol{\alpha} \cdot \nabla+\beta\left(M+V_{s}(r)\right)+V_{v}(r)\right] \mathcal{U}_{\alpha}=E_{\alpha} \mathcal{U}_{\alpha}
$$

and

$$
\begin{aligned}
\left(-\nabla^{2}+m_{\sigma}^{2}\right) \sigma & =-g_{\sigma} \rho_{s}(r)-g_{2} \sigma^{2}-g_{3} \sigma^{3}, \\
\left(-\nabla^{2}+m_{\omega}^{2}\right) \omega^{0} & =g_{\omega} \rho_{v}(r), \\
\left(-\nabla^{2}+m_{\rho}^{2}\right) \rho_{3}^{0} & =g_{\rho} \rho_{3}(r), \\
-\nabla^{2} A^{0} & =e \rho_{p}(r),
\end{aligned}
$$

where

$$
V_{s}=g_{\sigma} \sigma, \quad V_{v}=g_{\omega} \omega^{0}+g_{\rho} \tau_{3} \rho_{3}^{0}+e \frac{1+\tau_{3}}{2} A^{0},
$$

are the scalar and vector potentials, and

$$
\rho_{s}=\langle\bar{\psi} \psi\rangle, \rho_{v}=\left\langle\bar{\psi} \gamma^{0} \psi\right\rangle, \rho_{3}=\left\langle\bar{\psi} \gamma^{0} \tau_{3} \psi\right\rangle, \rho_{p}=\left\langle\bar{\psi} \gamma^{0} \frac{1+\tau_{3}}{2} \psi\right\rangle,
$$

the scalar, vector, isovector (third component) and proton densities. The expectation values are taken in the nuclear ground state, constructed by putting $Z$ protons and $N$ neutrons, $(Z+N=A)$, in the lowest particle states with $\tau_{3}$ equal to +1 and -1 , respectively, obtained by solving the Dirac equation (4). This yields the following results for the densities

$$
\begin{aligned}
\rho_{s} & =\sum_{\alpha=1}^{A} \overline{\mathcal{U}}_{\alpha} \mathcal{U}_{\alpha}, \rho_{v}=\sum_{\alpha=1}^{A} \overline{\mathcal{U}}_{\alpha} \gamma^{0} \mathcal{U}_{\alpha} \\
\rho_{3} & =\sum_{\alpha=1}^{A} \overline{\mathcal{U}}_{\alpha} \gamma^{0} \tau_{3} \mathcal{U}_{\alpha}, \rho_{p}=\sum_{\alpha=1}^{A} \overline{\mathcal{U}}_{\alpha} \gamma^{0} \frac{1+\tau_{3}}{2} \mathcal{U}_{\alpha} .
\end{aligned}
$$


The set of eqs. (4)-(8) should be solved self-consistently.

Alternatively, the RMFT equations could have been obtained by extremization of an energy functional built from eq. (1) and involving only valence nucleons and classical photon and meson fields. This density functional approach is sometimes preferred when working strictly within the context of an effective field theory, since it becomes clear in this case that much of the effects of vacuum dynamics and of certain many-body correlations beyond the Hartree level are absorbed in the renormalized coupling constants in the Lagrangian, when fitted to experimental data [5, 6].

The RRPA equations have been derived several times in the literature and applied, both to nuclear matter [16, and to finite nuclei [17]-21]. For our purpose here, they can be obtained from the equations of motion formalism of Rowe [22]. One introduces charge-exchange excitation operators of the form [23, 24, 25]

$$
O_{J \lambda}^{\dagger}=\sum_{p \bar{n}} X_{p \bar{n}}^{J \lambda}\left(a_{p}^{\dagger} a_{\bar{n}}\right)_{J}-\sum_{n \bar{p}} Y_{n \bar{p}}^{J \lambda}\left(a_{\bar{p}}^{\dagger} a_{n}\right)_{J}
$$

where $p$ and $\bar{p}$ ( $n$ and $\bar{n}$ ) label unoccupied and occupied proton (neutron) positive-energy, single-particle RMFT states. $J$ is the total angular momentum, and $\lambda$ runs from 1 to $\pi+\nu$, with $\pi$ and $\nu$ being the number of $p \bar{n}$ and $n \bar{p}$ excitations, i.e., those having $\sum_{p \bar{n}}\left|X_{p \bar{n}}^{J \lambda}\right|^{2}-\sum_{n \bar{p}}\left|Y_{n \bar{p}}^{J \lambda}\right|^{2}$ equal to +1 and -1 , respectively. Following the standard procedure one arrives at the RRPA equations

$$
\left(\begin{array}{cc}
\mathcal{A}^{J} & \mathcal{B}^{J} \\
\mathcal{B}^{J \dagger} & \mathcal{D}^{J}
\end{array}\right)\left(\begin{array}{c}
X^{J \lambda} \\
Y^{J \lambda}
\end{array}\right)=\omega_{J \lambda}\left(\begin{array}{c}
X^{J \lambda} \\
-Y^{J \lambda}
\end{array}\right) .
$$

The submatrices are given in terms of the residual interaction $V$ by

$$
\begin{aligned}
& \mathcal{A}_{p \bar{n}, p^{\prime} \bar{n}^{\prime}}^{J}=\left(E_{p}-E_{\bar{n}}\right) \delta_{p p^{\prime}} \delta_{\bar{n} \bar{n}^{\prime}}+\left\langle\left(p \bar{n}^{-1}\right)_{J}|V|\left(p^{\prime} \bar{n}^{\prime-1}\right)_{J}\right\rangle, \\
& \mathcal{D}_{n \bar{p}, n^{\prime} \bar{p}^{\prime}}^{J}=\left(E_{n}-E_{\bar{p}}\right) \delta_{n n^{\prime}} \delta_{\bar{p} \bar{p}}+\left\langle\left(n \bar{p}^{-1}\right)_{J}|V|\left(n^{\prime} \bar{p}^{\prime-1}\right)_{J}\right\rangle, \\
& \mathcal{B}_{p \bar{n}, n^{\prime} \bar{p}^{\prime}}^{J}=(-)^{J+M_{J}}\left\langle\left(p \bar{n}^{-1}\right)_{J M_{J}}\left(n^{\prime} \bar{p}^{\prime-1}\right)_{J,-M_{J}}|V|\right\rangle .
\end{aligned}
$$

The excitation energies $E_{J \lambda_{+}}^{+}\left(\lambda_{+}=1, \cdots \pi\right)$ in the odd-odd $(N-1, Z+1)$ nucleus (measured from the target ground state) are the highest $\pi$ eigenvalues $\omega_{J \lambda}$ in (10). In the same way the excitation energies $E_{J \lambda_{-}}^{-}\left(\lambda_{-}=1, \cdots \nu\right)$ in the $(N+1, Z-1)$ nucleus are the remaining $\nu$ eigenvalues $\omega_{J \lambda}$ taken with 
opposite sign. The corresponding total transition strengths are:

$$
\begin{aligned}
& S_{J}^{+}=\sum_{\lambda_{+}}\left|\sum_{p \bar{n}} X_{p \bar{n}}^{J \lambda_{+} *}\left\langle p \| w_{J}|| \bar{n}\right\rangle+\sum_{n \bar{p}} Y_{n \bar{p}}^{J \lambda_{+} *}\left\langle\bar{p}\left\|w_{J}\right\| n\right\rangle\right|^{2}, \\
& S_{J}^{-}=\sum_{\lambda_{-}}\left|\sum_{n \bar{p}} Y_{n \bar{p}}^{J \lambda_{-}}\left\langle\bar{p} \| w_{J}|| n\right\rangle+\sum_{p \bar{n}} X_{p \bar{n}}^{J \lambda_{-}}\left\langle p|| w_{J} \| \bar{n}\right\rangle\right|^{2},
\end{aligned}
$$

where $w_{0}=1$ and $w_{1}=\boldsymbol{\sigma}$. They fulfill the well known sum rules:

$$
S_{J}^{+}-S_{J}^{-}=(2 J+1)(N-Z) .
$$

For a self-consistent calculation, $V$ must be obtained from the same Lagrangian, given in eq. (11), which was used for the mean field part. Also, to be compatible with RMFT, one must consider only the direct terms in eq. (11), leading, in fact, to a ring approximation. Hence, only the isovector mesons contribute to isovector excitations like IAS and GTR. Furthermore, when the instantaneous approximation is made, the interaction, $V=V_{\pi}+V_{\rho}$, reads

$$
\begin{aligned}
& V_{\pi}(1,2)=-\left(\frac{f_{\pi}}{m_{\pi}}\right)^{2} \boldsymbol{\tau}_{1} \cdot \boldsymbol{\tau}_{2}\left(\boldsymbol{\sigma}_{1} \cdot \nabla_{1} \boldsymbol{\sigma}_{2} \cdot \nabla_{2}\right) Y\left(m_{\pi}, r\right), \\
& V_{\rho}(1,2)=g_{\rho}{ }^{2} \boldsymbol{\tau}_{1} \cdot \boldsymbol{\tau}_{2}\left(1-\boldsymbol{\alpha}_{1} \cdot \boldsymbol{\alpha}_{2}\right) Y\left(m_{\rho}, r\right),
\end{aligned}
$$

with $r=\left|\boldsymbol{r}_{1}-\boldsymbol{r}_{2}\right|$ and $Y(m, r)=\exp (-m r) /(4 \pi r)$.

We performed our numerical calculations taking ${ }^{48} \mathrm{Ca},{ }^{90} \mathrm{Zr}$ and ${ }^{208} \mathrm{~Pb}$ as the target nuclei. To get a discretized set of single-particle RMFT states, we solved eqs. (4)-(8) by expanding the different fields in truncated harmonic oscillator bases, following the method of P. Ring and collaborators as described in ref. [7], where the details can be found. For the RMFT parameters we chose the set NL1, which is widely used for nuclear ground state calculations [1], 7, 9]. The RRPA equations were solved for $J^{\pi}=0^{+}$and $1^{+}$states in a model-space including only $0 \hbar \Omega$ and $2 \hbar \Omega$ excitations. Furthermore, since the continuum is not well represented by an expansion in a harmonic oscillator basis, we tried to avoid it as much as possible. To this end, we included only those RMFT single-particle states that are bound at least for neutrons. (Of course, for consistency, we had to include the corresponding states for protons, even when unbound.) That this model-space is not too restricted is 
attested by the fact that, in all the cases considered here, the sum rule (13) was obeyed at the level of $99.5 \%(92.8 \%)$ or better for the IAS (GTR).

Our results for the IAS of ${ }^{208} \mathrm{~Pb}$ give an excitation energy (always measured from the target ground state) of $18.6 \mathrm{MeV}$ and a strength of $99 \%$ of the sum-rule prediction of $(N-Z)$, which compare well with the experimental values of $18.8 \mathrm{MeV}$ and $\sim 100 \%$, as quoted in [27]. These results are of the same quality as those of Colò et al. [14], who perform a nonrelativistic calculation that is also self-consistent, in the sense that they use the same interaction (Skyrme force) for the excitations as for the target ground state and single-particle basis.

Since the GTR's are rather broad, having experimental widths of $\sim$ $4 \mathrm{MeV}$, the theoretical results we report here correspond to a smoothed strength function, constructed by replacing the spikes in the RRPA strength distribution by Lorentzian peaks of conveniently chosen width. The excitation energy and strength of the GTR are then obtained by fitting a Lorentzian to the main peak in this strength function. When the interaction (14) is used we get that the excitation energy and the strength of the GTR in ${ }^{208} \mathrm{~Pb}$ are, respectively, $\sim 6 \mathrm{MeV}$ and $56 \%$ of the Ikeda sum-rule, while the corresponding experimental values are $19.2 \mathrm{MeV}$ and $60-70 \%$ [27]. Therefore, as it stands, the relativistic calculation would underestimate the GTR by more than $10 \mathrm{MeV}$, putting it much lower than the IAS, in blatant opposition to experiment. The nonrelativistic calculation of Colò et al. does much better, though it overestimates the excitation energy by $2-3 \mathrm{MeV}$.

It is still possible, however, to improve the GTR results in our calculation, without losing the self-consistency between the excitation and the mean field parts. In fact, when dealing with the pion contribution, we might follow the general practice and eliminate the contact term, which comes from the derivative (pseudovector) coupling and is thought to be suppressed by the short-range correlations between nucleons. (As such it should disappear when a form factor is used to account for finite nucleon and meson sizes.) More, to reproduce the energetics of the GTR within a nonrelativistic calculation based on $\pi$ - and $\rho$ - exchanges, Krewald et al. [12 were forced to add a zero-range Landau-Migdal interaction of adjustable strength. In our case this would correspond to gauge the contact term in the bare $\pi$-exchange 
interaction, given by (14), or equivalently to add the term

$$
\delta V_{\pi}(1,2)=g^{\prime}\left(\frac{f_{\pi}}{m_{\pi}}\right)^{2} \boldsymbol{\tau}_{1} \cdot \boldsymbol{\tau}_{2} \boldsymbol{\sigma}_{1} \cdot \boldsymbol{\sigma}_{2} \delta\left(\boldsymbol{r}_{1}-\boldsymbol{r}_{2}\right),
$$

which for $g^{\prime}=1 / 3$ completely cancels the contact part in (14). This term was found to play an important role in the RRPA calculation of unnatural parity states in ${ }^{16} \mathrm{O}$ made by Blunden and McCorquodale [17], who used the purely phenomenological value $g^{\prime}=0.7$, in analogy to nonrelativistic calculations. Since we are neglecting exchange contributions, $\delta V_{\pi}$ has no effect on the IAS, but is of overwhelming importance for the GTR, as illustrated in Fig. 11 for the case of ${ }^{208} \mathrm{~Pb}$. One sees that the theoretically justified coupling $g^{\prime}=1 / 3$ leads to better results than the bare interaction $\left(g^{\prime}=0\right)$. Yet, the agreement with experiment is only achieved with $g^{\prime} \cong 0.7$. Thus, we found it necessary to incorporate $\delta V_{\pi}$, with $g^{\prime}=0.7$, into the residual interaction.

The results obtained in our calculation, with this choice of residual interaction, for the IAS and GTR, excited from the closed-shell nuclei ${ }^{48} \mathrm{Ca}$, ${ }^{90} \mathrm{Zr}$ and ${ }^{208} \mathrm{~Pb}$, are summarized in Table 11. One can see that they are of the same quality as those obtained in similar nonrelativistic calculations [14]. The agreement with experiment is very good for the excitation energies of both resonances, but less so for the strengths, specially for the GTR. This was already expected, since our model does not include either $2 \mathrm{p}-2 \mathrm{~h}$ or $\Delta$-h excitations, and therefore is unable to reproduce the quenching of the GTR. For similar reasons, and also because we do not treat the continuum properly, we get very little strength at higher excitation energies beyond the GTR peak. In the case of ${ }^{90} \mathrm{Zr}$, for instance, our calculated strength function is decaying well below $0.45 \mathrm{MeV}^{-1}$ already for $\sim 25 \mathrm{MeV}$ excitation, while the experimental one [29] stays at about this value up to $\sim 60 \mathrm{MeV}$.

We conclude that both the IAS and the GTR excited from closed shell nuclei can be well reproduced in the context of quantum hadrodynamics, with the ground state parametrization for the $\sigma, \omega$ and $\rho$ mesons, and neglecting the exchange contributions. For the GTR, however, the pion with pseudovector coupling to the nucleon also must be included, and it is essential to use for the strength $g^{\prime}$ of the contact term $\delta V_{\pi}$ a value close to that used in similar nonrelativistic calculations. An alternative approach, to account for the energies of the GTR's, might be the mixed coupling of the rho meson to the nucleon. Yet, such a parametrization for ground state properties is available only in the Hartree-Fock approximation [10]. 
The authors wish to thank Peter Ring for enlightening discussions on the general topic of RMFT calculations and for giving them access to his spherical RMFT code and assistance in its usage. The work of C.D.C. was supported by CAPES (Brazil). A.P.G. and F.K. acknowledge partial financial support by ICTP (Trieste), CONICET (Argentina) and CLAF (Latin America).

\section{References}

[1] J. Meng and P. Ring, Phys. Rev. Lett. 77 (1996) 3963; D. Vretenar, G.A. Lalazissis and P. Ring, nucl-th/9712068 (submitted to Phys. Rev. C); G.A. Lalazissis, D. Vretenar, W. Pöschl and P. Ring, nucl-th/9710013 (submitted to Nucl. Phys. A) and nucl-th/9709020 (submitted to Phys.

Lett. B); W. Poeschl, D. Vretenar, G.A. Lalazissis and P. Ring, nuclth/9709027 (to appear in Phys. Rev. Lett.)

[2] B. D. Serot and J. D. Walecka, Adv. Nucl. Phys. 16 (1986) 1.

[3] J.D. Walecka, Theoretical Nuclear and Subnuclear Physics (Oxford University Press, New York, 1995)

[4] P. Ring, Prog. Part. Nucl. Phys. 37 (1996) 193.

[5] B. D. Serot and J. D. Walecka, Int. J. Mod. Phys. E 6 (1997) 515.

[6] R. J. Furnstahl, H.-B. Tang and B. D. Serot, Phys. Rev. C 52 (1995) 1368; R. J. Furnstahl, B. D. Serot and H.-B. Tang, Nucl. Phys. A 598 (1996) 539; A 618 (1997) 446.

[7] Y. K. Gambhir, P. Ring and A. Thimet, Ann. Phys. (N.Y.) 198 (1990) 132.

[8] J. Boguta and A. R. Bodmer, Nucl. Phys. A 292 (1977) 413.

[9] P.G. Reinhard, M. Rufa, J. Maruhn, W. Greiner and J. Friedrich, Z. Phys. A 323 (1986) 13.

[10] A. Bouyssy, J.F. Mathiot, N. Van Giai and S. Marcos, Phys. Rev. C 36 (1987) 380 . 
[11] R. Machleidt, Adv. Nucl. Phys. 19 (1989) 189.

[12] S. Krewald, F. Osterfeld, J. Speth and G. E. Brown, Phys. Rev. Lett. 46 (1981) 103.

[13] F. Osterfeld, Rev. Mod. Phys. 64 (1992) 491.

[14] G. Colò, N. Van Giai, P. F. Bortignon and R. A. Broglia, Phys. Rev. C 50 (1994) 1496.

[15] N. Dinh Dang, A. Arima, T. Suzuki and S. Yamaji, Phys. Rev. Lett. 79 (1997) 1638.

[16] H. Kurasawa and T. Suzuki, Nucl. Phys. A 445 (1985) 685.

[17] P. G. Blunden and P. McCorquodale, Phys. Rev. C 38 (1988) 1861.

[18] M. L'Huillier and N. Van Giai, Phys. Rev. C 39 (1989) 2022.

[19] J. R. Shepard, E. Rost and J. A. McNeil, Phys. Rev. C 40 (1989) 2320.

[20] J. F. Dawson and R. J. Furnstahl, Phys. Rev. C 42 (1990) 2009.

[21] Z. Ma, N. Van Giai, H. Toki and M. L'Huillier, Phys. Rev. C 55 (1997) 2385; Z. Ma, H. Toki and N. Van Giai, Nucl. Phys. A 627 (1997) 1.

[22] D.J. Rowe, Nuclear Collective Motion (Methuen, London, 1970).

[23] D. J. Rowe and C. Ngo-Trong, Rev. Mod. Phys. 47 (1975) 471.

[24] A. M. Lane and J. Martorell, Ann. Phys. (N.Y.) 129 (1980) 273.

[25] E. Bauer, F. Krmpotić and K. Nakayama, Nucl. Phys. A 485 (1988) 46.

[26] D. J. Horen et al., Phys. Lett. 95B (1980) 27.

[27] H. Akimune et al., Phys. Rev. C 52 (1995) 604.

[28] D. E. Bainum et al., Phys. Rev. Lett. 44 (1980) 1751.

[29] T. Wakasa et al., Phys. Rev. C 55 (1997) 2909.

[30] B. D. Anderson et al., Phys. Rev. C 31 (1985) 1161. 
Table 1: RRPA results for excitation energies and strengths of chargeexchange resonances calculated with isovector interaction including a Landau-Migdal-type contact term of intensity $g^{\prime}=0.7$, as described in the text. Experimental values are given for comparison.

\begin{tabular}{|c|c|c|c|c|}
\hline \multirow{2}{*}{$\begin{array}{l}\text { Parent } \\
\text { and } \\
\text { resonance }\end{array}$} & \multicolumn{2}{|r|}{ RRPA } & \multicolumn{2}{|c|}{ Experiment } \\
\hline & $\begin{array}{l}\text { Energy } \\
{[\mathrm{MeV}]}\end{array}$ & $\begin{array}{c}\text { Strength } \\
{[\% \text { of sum rule] }}\end{array}$ & $\begin{array}{l}\text { Energy } \\
{[\mathrm{MeV}]}\end{array}$ & $\begin{array}{c}\text { Strength } \\
\text { [\% of sum rule] }\end{array}$ \\
\hline \multicolumn{5}{|l|}{${ }^{208} \mathrm{~Pb}$} \\
\hline IAS & 18.6 & 99 & $18.83 \pm 0.02$ & $\sim 100$ \\
\hline GTR & 18.9 & 80 & $19.2 \pm 0.2$ & $60-70$ \\
\hline \multicolumn{5}{|l|}{${ }^{90} \mathrm{Zr}$ 田 } \\
\hline IAS & 11.9 & 101.3 & $12.0 \pm 0.2$ & $\sim 100$ \\
\hline GTR & 16.0 & 80 & $15.6 \pm 0.3$ & 28 \\
\hline \multicolumn{5}{|l|}{${ }^{48} \mathrm{Caf}$} \\
\hline IAS & 7.48 & 100.4 & 7.17 & $\sim 100$ \\
\hline GTR & 11.0 & 82 & $\sim 10.5$ & 35 \\
\hline
\end{tabular}

${ }^{a}$ Experimental values taken from Refs. 26, 27.

${ }^{b}$ Experimental values taken from Refs. [28, 29].

${ }^{c}$ Experimental values taken from Ref. [30]. 
Figure 1: Theoretical Gamow-Teller strength distribution for the parent nucleus ${ }^{208} \mathrm{~Pb}$ obtained with an isovector interaction including a LandauMigdal-type contact term of strength $g^{\prime}=0,1 / 3$ and 0.7 . The spikes give the raw RRPA results and the continuous curve, the strength function smoothed out as explained in the text. The strength function for the resonance peak extracted from experiment [27 is drawn in dotted line. 